way or another, could address the conference.

Even some 'alternative' groups could be heard in these sessions. At the same time, as the four sessions were meeting, an 'Environmental Forum' was running in Stockholm with papers and discussions by a great number of specialists in different fields.

The Stockholm conference was an experiment. An experiment in communication. It was clear that environmental problems of highly industrialized states are completely different from the problems of developing countries. For the latter, hunger, disease and low standard of living are the important factors which determine the environment that people have to live in; whereas high production, overconsumption, pollution, etc., determine the environmental problems of the industrialized countries. It was also clear that at such an international conference of a political nature, war and armament would be brought forward as environmental issues. It could hardly be denied that tests of nuclear weapons in the atmosphere, and defoliation, were as debatable as sources of air, water and soil contamination as the release of mercury in rivers, over-use of insecticides and ocean dumping. In spite of all the differences, the delegations never- theless noticed that most environmental problems may have aspects which have a common basis for which international co-operation would be necessary and possible in order to solve them. The 'action plan for the human environment' was, with a number of alterations, accepted. The governments agreed on a 'Declaration on the Human Environment'. That these agreements were possible proved that the communications-experiment was successful.

\section{After the Conference}

The decisions of the Stockholm Conference are to be confirmed by the next General Assembly of the United Nations and then the work can be started. From all the plans to be carried out, I only mention the installation of a world-wide measuring system. This will comprise 10 'baseline' stations in rural areas far from all sources of pollution to register longterm atmospheric and climatic changes. More than 100 stations of more regional scope will monitor the composition of the atmosphere. This system, which forms part of 'Earthwatch' has to provide data from which proliferation and sources of pollution can be studied.

Physicists and environmental problems Physics and physicists will be faced more and more with the necessity to contribute to the solutions of the world's major and crucial problems. Environmental research is badly needed. In the subject area studies, we find it stated many times that our knowledge on the environment is too fragmentary. Effective control measures are hampered by the gaps in our knowledge. On the other hand, many technological problems are present or coming up for which no solutions exist.

Better, reliable, simple, fast, sensitive and accurate monitors are needed to measure and study the behaviour of pollutants in water, air soil and food. Studies are needed of such environmental systems as the atmosphere and the energy balance of the earth. Studies on more careful use of earth resources are required. Research and development is necessary to face the problems of energy-supplies, acoustics, traffic and communications.

National research programmes will be started in international co-operation, in which physicists from all specializations can contribute in field measurements, data analysis, laboratory experiments and theory.

In my opinion, the European Physical Society could play an important role in the exploration of this new dimension of physics.

\title{
Fourth Conference on Atomic Spectroscopy
}

Amsterdam, The Netherlands, 11-14 July 1972

\section{Organized by the European Group for Atomic Spectroscopy (Specialized Division of EPS)}

With this 4th Conference, EGAS continued the series of annual conferences that started in Orsay in 1969.

The University of Amsterdam extended highly appreciated hospitality to the Conference in its Mathematics Lecture Building. The Group for Atomic Spectroscopy of the Zeeman Laboratorium actually carried out the organization.

In accordance with the aims of EGAS ("..., to facilitate contacts between members, ..., to stimulate the interest of young physicists in the field of atomic spectroscopy, ...") the conferenc fee was kept low (Dfl 35.-) and no restrictions were imposed, neither on the number, nor on the subject, of contributed papers that could be accepted.

Indeed, the Conference attracted a large number of young spectroscopists. Altogether 225 participants were registered, mainly from France, Fed. Rep. Germany, Netherlands, Sweden and U.K, but also from Bulgaria, Denmark, Israel, Italy, Poland, Spain, Switzerland, USA and Yugoslavia.

As in previous years, the programme was devoted to contributed papers, invited papers, round-table discussions and laboratory visits. The large number of submitted papers $(88$, each to be presented within 15 minutes) made it necessary to place two-thirds of them in parallel sessions.

Three invited papers each reviewed an interesting topic within the scope of EGAS :

1 UItraviolet stellar spectrophotometry by $C$. de Jager (Utrecht)

2 Analysis of atomic spectra ; the present situation and the future by $B$. Edlen (Lund)

3 Dye lasers and some of their applications by $B$. Bölger (Eindhoven).

Round-table discussions were organized on the following topics, suggested by individual participants and by the subjects of submitted papers :
1 Problems connected with spectra in the vacuum UV

2 Analysis of spectra

3 Lasers and their applications

4 Hanle effect and level crossing

5 Hyperfine structure and isotope shift ; measurement and interpretation

6 Theory and experiment of hyperfine structure and the evaluation of nuclear moments.

Summaries of discussions will be published in the EGAS abstracts lists.

The final paper was given by $P$. Jacquinot (Orsay) who discussed in detail the possibilities and limitations of high-resolution Fourier spectrometry and who announced the facilities of Laboratoire Aimé Cotton in this field - an idea that certainly is worth-while - to be considered and to be followed by other laboratories.

As many of the papers were of a preliminary character, no proceedings will be published.

E. Meinders 